\title{
A Finite Element Method for the Stationary Stokes Equations Using Trial Functions Which Do Not Have to Satisfy $\operatorname{div} v=0$
}

\author{
By Richard S. Falk*
}

\begin{abstract}
By adding a term to the variational equations, we derive a new finite element method for the stationary Stokes equations which eliminates the $\operatorname{div} v=0$ restriction on the trial functions. The method is described using continuous piecewise linear functions, and the optimal $O(h)$ order of convergence estimate is derived for the error in the $H^{1}(\Omega)$ norm.
\end{abstract}

1. Introduction. A major obstacle in using finite element methods to approximate the solution of Stokes equations is the construction of trial functions satisfying either zero divergence or some other condition which approximates it. We present in this note a method for avoiding this problem by adding a term to the variational equations. The weighted method produced is then shown to give the optimal $O(h)$ order of convergence estimate when applied using continuous, piecewise linear trial functions.

For simplicity we confine ourselves here to the approximation of the stationary Stokes equations, i.e.

Problem (P). Find $\mathbf{u}=\left(u_{1}, \ldots, u_{N}\right)$ and $p$ defined on $\Omega$ such that

$$
-\nu \Delta \mathbf{u}+\operatorname{grad} p=\mathbf{f} \text { in } \Omega, \quad \operatorname{div} \mathbf{u}=0 \quad \text { in } \Omega, \quad \mathbf{u}=\mathbf{0} \quad \text { on } \partial \Omega,
$$

where $\mathbf{u}$ is the fluid velocity, $p$ is the pressure, $\mathbf{f}$ are the body forces per unit mass and $\nu>0$ is the dynamic viscosity.

For other finite element methods for this problem, see the excellent bibliography in Temam [7]. Some other recent methods not listed there are those of [1]-[5].

2. Notation. We shall consider Problem (P) in the case where $\Omega$ is a bounded convex domain in $\mathbf{R}^{N}$ with $C^{2}$ boundary. The results of this paper will also hold for $\Omega$ a convex polygon.

Denote by $(u, v)$ the $L^{2}(\Omega)$ inner product and by $\|v\|_{0}$ the norm $(v, v)^{1 / 2}$. For $m$ a nonnegative integer we denote by $H^{m}(\Omega)\left(H_{0}^{m}(\Omega)\right)$ the completion of $C^{\infty}(\bar{\Omega})$ $\left(C_{0}^{\infty}(\Omega)\right)$ in the norm

$$
\|v\|_{m}=\left(\sum_{|\alpha| \leqslant m}\left\|D^{\alpha} v\right\|_{0}^{2}\right)^{1 / 2} .
$$

For $m$ a negative integer we define $H^{m}(\Omega)$ as the completion of $C^{\infty}(\bar{\Omega})$ with respect to the norm

Received September 16, 1975; revised December 15, 1975.

AMS (MOS) subject classifications (1970). Primary 35J20, 41 A15, 41 A25, 65N30.

* This work was supported by NSF Grant MPS 74-05795. 


$$
\|v\|_{m}=\sup _{w \in C^{\infty}(\bar{\Omega})} \frac{(v, w)}{\|w\|_{-m}} \text {. }
$$

We will also be using the inner product in $H^{m}(\Omega)$ which we denote by $(u, v)_{m}$.

For vector valued functions $\mathbf{v}=\left(v_{1}, \ldots, v_{N}\right)$ we let $\left[H^{m}(\Omega)\right]^{N}$ be the space of $\mathbf{v}$ with components $v_{i} \in H^{m}(\Omega)$. The scalar product and norm in $\left[H^{m}(\Omega)\right]^{N}$ are given by

$$
(\mathbf{u}, \mathbf{v})_{m}=\sum_{i=1}^{N}\left(u_{i}, v_{i}\right)_{m} \text { and }\|\mathbf{v}\|_{m}=\left(\sum_{i=1}^{N}\left\|v_{i}\right\|_{m}^{2}\right)^{1 / 2}, \text { respectively }
$$

Finally, for convenience we introduce the bilinear form

$$
a(\mathbf{u}, \mathbf{v})=\nu \sum_{k=1}^{N} \sum_{l=1}^{N} \int_{\Omega} \frac{\partial u_{k}}{\partial x_{l}} \frac{\partial v_{k}}{\partial x_{l}} d x
$$

defined on $\left[H_{0}^{1}(\Omega)\right]^{N} \times\left[H_{0}^{1}(\Omega)\right]^{N}$ and the corresponding norm $\|\mathbf{u}\|_{E}^{2}=a(\mathbf{u}, \mathbf{u})$.

3. Approximate Problem and Error Estimates. We begin our discussion with a statement of a regularity result for the solution of Problem (P) and a description of the subspaces we will use in its approximation.

Lemma 1 (Temam [7]). If $\Omega$ is an open bounded set of class $C^{2}$ and if $\mathbf{f}$ belongs to $\left[L^{2}(\Omega)\right]^{N}$, then the solution $\mathbf{u}, p$ of Problem $(\mathrm{P})$ satisfies $\mathbf{u} \in\left[H^{2}(\Omega)\right]^{N}$, $p \in H^{1}(\Omega)$ and $\|\mathbf{u}\|_{2}+\|p\|_{1} \leqslant C(\nu, \Omega)\|\mathbf{f}\|_{0}$. (We have assumed that $p$ has been normalized so that $\int_{\Omega} p d x=0$.)

We remark that a similar result has been proved by Kellogg and Osborn in [6] for $\Omega$ a polygonal domain.

Since $\Omega$ is assumed convex, the finite dimensional subspaces we will use in the approximation of Problem (P) can be described as follows. Let $h, 0<h<1$, be a parameter, and for each value of $h$, let $\Omega_{h}$ be a polygon inscribed in $\Omega$ with all its vertices lying on $\partial \Omega$ and each side of the polygon of length less than or equal to $h$. Let $\Delta_{h}\left(\Omega_{h}\right)$ be a regular triangularization of $\Omega_{h}$ and $\Delta_{h}(\Omega)$ an extension of the triangularization $\Delta_{h}\left(\Omega_{h}\right)$ to cover $\Omega$. Set $V_{h}=\left\{v_{h}: v_{h}\right.$ is continuous on $\Omega$ and linear on each triangle of $\left.\Delta_{h}(\Omega)\right\}$ and $V_{h}^{0} \stackrel{\circ}{=}\left\{v_{h} \in V_{h}: v_{h}=0\right.$ in $\left.\bar{\Omega}-\Omega_{h}\right\}$.

Then the following approximation results are satisfied by $V_{h}$ and $V_{h}^{0}$ for some constant $C$ independent of $u$ and $h$.

For $u \in H^{2}(\Omega)$, there exists a $v_{h} \in V_{h}$ such that

$$
\left\|u,-v_{h}\right\|_{0}+h\left\|u-v_{h}\right\|_{1} \leqslant C h^{2}\|u\|_{2} .
$$

If $u \in H^{2}(\Omega) \cap H_{0}^{1}(\Omega)$, then (*) is satisfied for a $v_{h} \in V_{h}^{0}$.

From the point of view of approximation theoretic results like $(*)$, one might like to formulate an approximation scheme for Problem (P) of the form:

Problem $\left(\mathrm{P}_{h}^{\prime}\right)$. Find $\mathbf{u}_{h} \in\left[V_{h}^{0}\right]^{N}$ such that $a\left(\mathbf{u}_{h}, \mathbf{v}_{h}\right)+\gamma h^{-2}\left(\operatorname{div} \mathbf{u}_{h}, \operatorname{div} \mathbf{v}_{h}\right)_{-1}=$ (f, $\left.\mathbf{v}_{h}\right)$ for all $\mathbf{v}_{h} \in\left[V_{h}^{0}\right]^{N}(\gamma>0$ a constant).

For this type of scheme it follows in a very straightforward manner that

$$
\begin{aligned}
\left\|\mathbf{u}-\mathbf{u}_{h}\right\|_{E} & +h^{-1}\left\|\operatorname{div} \mathbf{u}_{h}\right\|_{-1} \\
& \leqslant C\left\{\left\|\mathbf{u}-\mathbf{v}_{h}\right\|_{E}+h\|p\|_{1}+h^{-1}\left\|\operatorname{div}\left(\mathbf{u}-\mathbf{v}_{h}\right)\right\|_{-1}\right\}
\end{aligned}
$$


for all $\mathbf{v}_{h} \in\left[V_{h}^{0}\right]^{N}$. Since $\left\|\operatorname{div}\left(\mathbf{u}-\mathbf{v}_{h}\right)\right\|_{-1} \leqslant\left\|\mathbf{u}-\mathbf{v}_{h}\right\|_{0}$, it follows immediately from (1) and (*) that $\left\|\mathbf{u}-\mathbf{u}_{h}\right\|_{E} \leqslant C h$.

The obvious trouble with this scheme is that ( $\left.\operatorname{div} u_{h}, \operatorname{div} v_{h}\right)_{-1}$ is not a computable quantity (in a practical sense). The central idea of this note is to show how this quantity can be replaced by a computable one, while still retaining the same $O(h)$ convergence rate.

To do so, we define a bilinear form $b(\mathbf{w}, \mathbf{z})=h^{2}(\operatorname{div} \mathbf{w}, \operatorname{div} \mathbf{z})+\left(Q_{h}(\mathbf{w}), \operatorname{div} \mathbf{z}\right)$ where $Q_{h}(\mathbf{w}) \in V_{h}$ satisfies

$$
\left(Q_{h}(w), \psi_{h}\right)_{1}=\left(\operatorname{div} w, \psi_{h}\right) \text { for all } \psi_{h} \in V_{h} .
$$

Using this form, we define another approximate problem as follows:

Problem $\left(\mathrm{P}_{h}\right)$. Find $\mathrm{u}_{h} \in\left[V_{h}^{0}\right]^{N}$ such that

(3) $a\left(\mathbf{u}_{h}, \mathbf{v}_{h}\right)+\gamma h^{-2} b\left(\mathbf{u}_{h}, \mathbf{v}_{h}\right)=\left(\mathbf{f}, \mathbf{v}_{h}\right)$ for all $\mathbf{v}_{h} \in\left[V_{h}^{0}\right]^{N} \quad(\gamma>0$ a constant $)$.

Then the main result is:

Theorem 1. Let $\mathrm{u}$ and $\mathrm{u}_{h}$ be the respective solutions of Problems $(\mathrm{P})$ and $\left(\mathrm{P}_{h}\right)$. Then if $\mathrm{f} \in\left[L^{2}(\Omega)\right]^{N}$, there exists a constant $C$ independent of $h$ and $\mathbf{f}$ such that

$$
\left\|\mathbf{u}-\mathbf{u}_{h}\right\|_{E}+\left\|\operatorname{div} \mathbf{u}_{h}\right\|_{0} \leqslant C h\|\mathbf{f}\|_{0} .
$$

In order to prove Theorem 1, we first establish some properties of the bilinear form $b(\mathbf{w}, \mathbf{z})$.

Lemma 2. If $\mathrm{w} \in\left[H^{1}(\Omega)\right]^{N}$, then

$$
\|\operatorname{div} \mathbf{w}\|_{-1}^{2} \leqslant C b(\mathbf{w}, \mathbf{w})=C\left[h^{2}\|\operatorname{div} \mathbf{w}\|_{0}^{2}+\left\|Q_{h}(\mathbf{w})\right\|_{1}^{2}\right]
$$

for some constant $C$ independent of $\mathbf{w}$.

Proof. Let $Q(\mathrm{w})$ be the solution in $H^{1}(\Omega)$ of $(Q(\mathrm{w}), \psi)_{1}=(\operatorname{div} \mathrm{w}, \psi)$ for all $\psi \in H^{1}(\Omega)$.

Then by a well-known regularity result for elliptic boundary value problems,

$$
Q(\mathrm{w}) \in H^{2}(\Omega) \text { and }\|Q(\mathrm{w})\|_{2} \leqslant C\|\operatorname{div} \mathbf{w}\|_{0}
$$

for some constant $C$ independent of $\mathbf{w}$. By (2),

$$
\left(Q(\mathbf{w})-Q_{h}(\mathbf{w}), \psi_{h}\right)_{1}=0 \quad \text { for all } \psi_{h} \in V_{h} \text {. }
$$

Hence,

Now

$$
\begin{array}{rlrl}
\left\|Q(w)-Q_{h}(w)\right\|_{1} & \leqslant\left\|Q(w)-\psi_{h}\right\|_{1} & \left(\text { for all } \psi_{h} \in V_{h}\right) \\
& \leqslant C h\|\operatorname{div} w\|_{0} \quad\left(\text { by }\left(^{*}\right) \text { and }(4)\right) .
\end{array}
$$

$$
\begin{aligned}
\|\operatorname{div} w\|_{-1} & =\|Q(w)\|_{1} \leqslant\left\|Q(w)-Q_{h}(w)\right\|_{1}+\left\|Q_{h}(w)\right\|_{1} \\
& \leqslant C h\|\operatorname{div} w\|_{0}+\left\|Q_{h}(w)\right\|_{1} .
\end{aligned}
$$

Hence,

$$
\begin{aligned}
\|\operatorname{div} w\|_{-1}^{2} & \leqslant C\left[h^{2}\|\operatorname{div} w\|_{0}^{2}+\left\|Q_{h}(\mathbf{w})\right\|_{1}^{2}\right] \\
& =C b(\mathbf{w}, \mathbf{w}) \quad\left(\text { since }\left\|Q_{h}(\mathbf{w})\right\|_{1}^{2}=\left(Q_{h}(\mathbf{w}), \operatorname{div} w\right)\right) .
\end{aligned}
$$


Remark. From Lemma (2) it easily follows that Problem $\left(\mathrm{P}_{h}\right)$ has a unique solution.

LEMMA 3.

$$
b(\mathbf{w}, \mathrm{z}) \leqslant \frac{1}{4} b(\mathrm{w}, \mathbf{w})+h^{2}\|\operatorname{div} \mathbf{z}\|_{0}^{2}+\|\operatorname{div} \mathbf{z}\|_{-1}^{2} .
$$

Proof.

$$
\begin{aligned}
b(\mathrm{w}, \mathrm{z}) & =h^{2}(\operatorname{div} \mathrm{w}, \operatorname{div} \mathrm{z})+\left(Q_{h}(\mathrm{w}), \operatorname{div} \mathrm{z}\right) \\
& \leqslant h^{2}\|\operatorname{div} \mathrm{w}\|_{0}\|\operatorname{div} \mathrm{z}\|_{0}+\left\|Q_{h}(\mathrm{w})\right\|_{1}\|\operatorname{div} \mathbf{z}\|_{-1} \\
& \leqslant 1 / 4 h^{2}\|\operatorname{div} \mathrm{w}\|_{0}^{2}+h^{2}\|\operatorname{div} \mathrm{z}\|_{0}^{2}+1 / 4\left\|Q_{h}(\mathrm{w})\right\|_{1}^{2}+\|\operatorname{div} \mathbf{z}\|_{-1}^{2} \\
& \leqslant 1 / 4 b(\mathrm{w}, \mathrm{w})+h^{2}\|\operatorname{div} \mathbf{z}\|_{0}^{2}+\|\operatorname{div} \mathbf{z}\|_{-1}^{2} .
\end{aligned}
$$

We now prove Theorem 1.

Proof. Since u solves Problem (P), it follows easily that

$$
a(\mathbf{u}, \mathbf{v})+\gamma h^{-2} b(\mathbf{u}, \mathbf{v})=(\mathbf{f}, \mathbf{v})-(\operatorname{grad} p, \mathbf{v}) \quad \text { for all } \mathbf{v} \in\left[H_{0}^{1}(\Omega)\right]^{N} .
$$

Since $u_{h}$ solves Problem $\left(\mathrm{P}_{h}\right)$, we may subtract Eq. (3) from the above to get

$$
a\left(\mathbf{u}-\mathbf{u}_{h}, \mathbf{v}_{h}\right)+\gamma h^{-2} b\left(\mathbf{u}-\mathbf{u}_{h}, \mathbf{v}_{h}\right)=-\left(\operatorname{grad} p, \mathbf{v}_{h}\right) \text { for all } \mathbf{v}_{h} \in\left[V_{h}^{0}\right]^{N} .
$$

It follows easily that

Now

$$
\begin{aligned}
& \left\|\mathbf{u}-\mathbf{u}_{h}\right\|_{E}^{2}+\gamma h^{-2} b\left(\mathbf{u}-\mathbf{u}_{h}, \mathbf{u}-\mathbf{u}_{h}\right) \\
& \quad=a\left(\mathbf{u}-\mathbf{u}_{h}, \mathbf{u}-\mathbf{v}_{h}\right)+\gamma h^{-2} b\left(\mathbf{u}-\mathbf{u}_{h}, \mathbf{u}-\mathbf{v}_{h}\right)-\left(\operatorname{grad} p, \mathbf{v}_{h}-\mathbf{u}_{h}\right) .
\end{aligned}
$$

$-\left(\operatorname{grad} p, \mathbf{v}_{h}-\mathbf{u}_{h}\right)=\left(p, \operatorname{div}\left(\mathbf{v}_{h}-\mathbf{u}_{h}\right)\right)=\left(p, \operatorname{div}\left(\mathbf{v}_{h}-\mathbf{u}\right)\right)+\left(p, \operatorname{div}\left(\mathbf{u}-\mathbf{u}_{h}\right)\right)$

$$
\begin{aligned}
\leqslant & \|p\|_{1}\left[\left\|\operatorname{div}\left(\mathbf{v}_{h}-\mathbf{u}\right)\right\|_{-1}+\left\|\operatorname{div}\left(\mathbf{u}-\mathbf{u}_{h}\right)\right\|_{-1}\right] \\
\leqslant & C\|p\|_{1}\left[\left\|\operatorname{div}\left(\mathbf{v}_{h}-\mathbf{u}\right)\right\|_{-1}+\left\{b\left(\mathbf{u}-\mathbf{u}_{h}, \mathbf{u}-\mathbf{u}_{h}\right)\right\}^{1 / 2}\right] \quad \text { (by Lemma 2) } \\
\leqslant & C h^{2}\|p\|_{1}^{2}+h^{-\dot{2}}\left\|\operatorname{div}\left(\mathbf{v}_{\dot{n}}-\mathbf{u}\right)\right\|_{-1}^{2} \\
& +1 / 4 \gamma h^{-2} b\left(\mathbf{u}-\mathbf{u}_{h}, \mathbf{u}-\mathbf{u}_{h}\right) \text { (using the arithmetic-geometric mean inequality). }
\end{aligned}
$$

By Lemma 3,

$$
\begin{aligned}
& \quad \gamma h^{-2} b\left(\mathbf{u}-\mathbf{u}_{h}, \mathbf{u}-\mathbf{v}_{h}\right) \\
& \quad \leqslant 1 / 4 \gamma h^{-2} b\left(\mathbf{u}-\mathbf{u}_{h}, \mathbf{u}-\mathbf{u}_{h}\right)+\gamma\left\|\operatorname{div}\left(\mathbf{u}-\mathbf{v}_{h}\right)\right\|_{0}^{2}+\gamma h^{-2}\left\|\operatorname{div}\left(\mathbf{u}-\mathbf{v}_{h}\right)\right\|_{-1}^{2},
\end{aligned}
$$

and using the Schwarz inequality,

$$
a\left(\mathbf{u}-\mathbf{u}_{h}, \mathbf{u}-\mathbf{v}_{h}\right) \leqslant 1 / 2\left\|\mathbf{u}-\mathbf{u}_{h}\right\|_{E}^{2}+1 / 2\left\|\mathbf{u}-\mathbf{v}_{h}\right\|_{E}^{2} .
$$

Hence, after collecting terms, we obtain

$$
\begin{aligned}
& \left\|\mathbf{u}-\mathbf{u}_{h}\right\|_{E}^{2}+\gamma h^{-2} b\left(\mathbf{u}-\mathbf{u}_{h}, \mathbf{u}-\mathbf{u}_{h}\right) \\
& \leqslant C\left[\left\|\mathbf{u}-\mathbf{v}_{h}\right\|_{E}^{2}+\left\|\operatorname{div}\left(\mathbf{u}-\mathbf{v}_{h}\right)\right\|_{0}^{2}+h^{-2}\left\|\operatorname{div}\left(\mathbf{u}-\mathbf{v}_{h}\right)\right\|_{-1}^{2}+h^{2}\|p\|_{1}^{2}\right] \\
& \quad \text { for all } \mathbf{v}_{h} \in\left[V_{h}^{0}\right]^{N} .
\end{aligned}
$$

Since $\left\|\operatorname{div}\left(\mathbf{u}-\mathbf{v}_{h}\right)\right\|_{-1} \leqslant\left\|\mathbf{u}-\mathbf{v}_{h}\right\|_{0}$, it follows immediately from (*) and Lemma 1 
that

$$
\left\|\mathbf{u}-\mathbf{u}_{h}\right\|_{E}+\sqrt{\gamma} h^{-1}\left\{b\left(\mathbf{u}-\mathbf{u}_{h}, \mathbf{u}-\mathbf{u}_{h}\right)\right\}^{1 / 2} \leqslant C h\|\mathbf{f}\|_{0}
$$

and hence, using the definition of $b(\mathbf{w}, \mathbf{z})$, that

$$
\left\|\mathbf{u}-\mathbf{u}_{h}\right\|_{E}+\left\|\operatorname{div} \mathbf{u}_{h}\right\|_{0} \leqslant C h\|\mathbf{f}\|_{0} .
$$

4. Final Comments. In the preceding we have presented a method for the approximation of the stationary Stokes equations which yields optimal $O(h)$ convergence using piecewise linear elements with no zero divergence restriction. For the elimination of the constraint and the advantage of using such simple finite elements, one must pay a price. In this case it is the added complexity of the linear system which must be solved.

Finally, we note that it is possible to formulate an optimal order method for finite elements of order $r>2$ by replacing the term $\gamma h^{-2} b\left(\mathbf{u}_{h}, \mathbf{v}_{h}\right)$ by

where

$$
\gamma \sum_{m=2}^{r} h^{2-2 m} b_{m}\left(\mathbf{u}_{h}, \mathbf{v}_{h}\right)
$$

$$
b_{m}(\mathbf{w}, \mathbf{z})=h^{2 m-2}(\operatorname{div} \mathbf{w}, \operatorname{div} \mathbf{z})+\left(Q_{h}^{m}(\mathbf{w}), \operatorname{div} \mathbf{z}\right)
$$

and $Q_{h}^{m}(\mathbf{w})$ satisfies

$$
\left(Q_{h}^{m}(\mathrm{w}), \psi_{h}\right)_{m-1}=\left(\operatorname{div} \mathbf{w}, \psi_{h}\right) \text { for all } \psi_{h} \in S_{h}^{m-1,2 m-2},
$$

where $S_{h}^{m-1,2 m-2}$ is a finite dimensional subspace of $H^{m-1}(\Omega)$ with the property that for $u \in H^{2 m-2}(\Omega)$, there exists $\psi_{h} \in S_{h}^{m-1,2 m-2}$ such that

$$
\left\|u-\psi_{h}\right\|_{m-1} \leqslant C h^{m-1}\|u\|_{2 m-2} .
$$

However, because the auxiliary problems (5) increase in order, this does not seem to be a practical approach.

Department of Mathematics

Rutgers University

New Brunswick, New Jersey 08903

1. M. CROUZEIX \& P. A. RAVIART, "Conforming and nonconforming finite element methods for solving the stationary Stokes equations. I," Rev. Française Automat. Informat. Recherche Opérationnelle Sér. Rouge, v. 7, 1973, pp. 33-75. MR 49 \# 8401.

2. R. S. FALK, "An analysis of the finite element method using Lagrange multipliers for the stationary Stokes equations," Math. Comp., v. 30, 1976, pp. 241-249.

3. R. S. FALK, "An analysis of the penalty method and extrapolation for the stationary Stokes equations," Advances in Computer Methods for Partial Differential Equations, R. Vichnevetsky (Editor), Proc. AICA Sympos., Lehigh Univ., June 1975, pp. 66-69.

4. R. S. FALK \& J. T. KING, "A penalty and extrapolation method for the stationary Stokes equations," SIAM J. Numer. Anal., v. 13, 1976.

5. P. JAMET \& P. A. RAVIART, Numerical Solution of the Stationary Navier-Stokes Equations by Finite Element Methods, Lecture Notes in Computer Science, Vol. 10 (Computing Methods in Applied Sciences and Engineering, Part 1, International Symposium, Versailles, Dec. 1973), J. L. Lions \& R. Glowinski (Editors), Springer-Verlag, New York, 1974, pp. 193-223.

6. B. KELLOGG \& J. OSBORN, A Regularity Result for the Stokes Problem in a Convex Polygon, Technical Note BN-804, Institute for Fluid Dynamics and Applied Mathematics, Univ. of Maryland, 1974.

7. R. TEMAM, On the Theory and Numerical Analysis of the Navier-Stokes Equations, Lecture Note \# 9, Univ. of Maryland, June 1973. 